\title{
RESEARCH LETTERS
}

\section{Caring for Melanoma Survivors with Self-detected Concerning Moles During COVID-19 Restricted Physician Access: A Cohort Study}

\author{
June K. Robinson, MD¹, Burkhard Jansen, MD² \\ ${ }^{1}$ Department of Dermatology, Northwestern University Feinberg School of Medicine, Chicago, IL \\ ${ }^{2}$ DermTech. Inc., La Jolla, CA
}

\section{ABSTRACT}

Background: Physician appointments for non-essential care ceased during COVID-19.

Objective: To pilot test a telehealth solution for patients to rule out melanomas and need for surgical biopsies based on genomic analyses of pigmented lesion samples obtained via adhesive patches.

Methods: Surveys assessed SSE anxiety. Under remote clinician guidance, patients or partners obtained samples using adhesive patches (DermTech, La Jolla, CA).

Results: SSE anxiety increased. Guided self-sampling led to molecular risk factor analyses in $7 / 7(100 \%)$ of cases compared to $9 / 10(90 \%)$ randomly selected physician-sampled control cases.

Conclusions: Adhesive patch self-sampling under remote physician guidance is a viable specimen collection option.

\section{INTRODUCTION}

Self-management of melanoma detection with skin self-examination (SSE) by melanoma survivors and other patients atrisk to develop melanoma depends on ready access to dermatologists when a concerning mole is detected. ${ }^{1}$ With the March 2020 Illinois stay at home order (COVID-19) inperson physician appointments for nonessential care ceased. Additionally, there is uncertainty about when regularly scheduled health care would resume. Dermatologists currently provide care under expanded telehealth benefits using electronic health record (EHR) portals for synchronous evisits. ${ }^{2}$ Despite the limited quality of storeforward images, ${ }^{3}$ physicians attempted to interpret patient's photographs and videos of concerning moles; however, improvements and objective assessments beyond image interpretation appear highly desirable.

The cohort study of melanoma survivors presented here assessed SSE anxiety prior to and during restricted physician access. It furthermore evaluated a telehealth support solution that enables patients to rule out melanomas and the need for surgical May 2020 Volume 4 Issue 3 
biopsies based on genomic analyses of pigmented lesion samples obtained noninvasively via adhesive patches. Patients applied adhesive patches to confirmed skin lesions suspicious for melanoma under remote guidance by their dermatologist (JKR). ${ }^{4}$ (DermTech, Inc. La Jolla, CA.)

\section{METHODS}

Melanoma survivors were trained to perform partner assisted SSE and completed periodic online surveys assessing SSE performance, identification of concerning moles, physician visits for moles, and biopsies of moles. ${ }^{5}$ SSE anxiety and benefit were assessed (Table 1).

In March 2020, melanoma survivors submitted photographs of concerning moles that had changed or had moles reviewed via FaceTime by their dermatologist, who determined if the mole was clinically suspicious for melanoma. For confirmed concerning moles, the dermatologist ordered adhesive patch skin sample collection kits (DermTech, La Jolla, CA) to be couriered to patients. Patients or their skin check partners obtained the samples under remote clinician guidance. Samples were returned by courier to DermTech for LINC00518 and PRAME genomic risk factor analyses via DermTech's Pigmented Lesion Assay (PLA). ${ }^{4}$ The dermatologist communicated test results and next steps to patients remotely. Subjects were interviewed about their mole selfsampling experience. The Institutional Review Board of Northwestern University approved the research. Melanoma survivors received $\$ 20$ for each survey and those who submitted a specimen received $\$ 50$.

\section{RESULTS}

There were 211 respondents among 258 eligible melanoma survivors (81.7\%). In the
Table 1. Skin self-examination induced anxiety^

\begin{tabular}{|c|c|c|}
\hline $\begin{array}{l}\text { SSE Anxiety } \\
\text { In recent months, ... }\end{array}$ & $\begin{array}{l}\text { Prior to } \\
\text { COVID-19 } \\
\text { Mean (SD) } \\
(n=258)\end{array}$ & $\begin{array}{l}\text { During } \\
\text { COVID-19 } \\
\text { Mean (SD) } \\
(\mathrm{n}=211)\end{array}$ \\
\hline $\begin{array}{l}\text { Checking for moles } \\
\text { caused me some } \\
\text { distress. }\end{array}$ & $1.2(0.3)$ & $3.1(0.2)^{*}$ \\
\hline $\begin{array}{l}\text { Checking for moles } \\
\text { made me very } \\
\text { concerned about } \\
\text { having a melanoma. }\end{array}$ & $1.9(0.4)$ & $4.3(0.6)^{*}$ \\
\hline $\begin{array}{l}\text { I felt fearful when I } \\
\text { checked my skin. }\end{array}$ & $1.3(0.2)$ & $2.1(0.9)$ \\
\hline $\begin{array}{l}\text { I found it hard to focus } \\
\text { on anything else other } \\
\text { than my anxiety when } \\
\text { I checked my skin. }\end{array}$ & $1.1(0.1)$ & $1.8(0.1)$ \\
\hline $\begin{array}{l}\text { My worries } \\
\text { overwhelmed me } \\
\text { when I checked my } \\
\text { skin. }\end{array}$ & $1.2(0.2)$ & $1.3(0.2)$ \\
\hline $\begin{array}{l}\text { I felt uneasy when I } \\
\text { checked my skin. }\end{array}$ & $1.4(0.3)$ & $3.4(1.5)^{*}$ \\
\hline $\begin{array}{l}\text { SSE benefit: } \\
\text { decision to seek } \\
\text { health care }\end{array}$ & & \\
\hline $\begin{array}{l}\text { I feel that checking my } \\
\text { moles has helped me } \\
\text { to better be able to } \\
\text { decide if a mole needs } \\
\text { to be checked by a } \\
\text { doctor. }\end{array}$ & $4.6(0.7)$ & $4.8(0.2)$ \\
\hline \multicolumn{3}{|c|}{$\begin{array}{l}{ }^{*} \chi^{2} p<.05 \\
\text { ^Likert scale } 1=\text { strongly disagree, } 3=\text { neutral, } 5=\text { strongly } \\
\text { agree. Adapted from PROMIS Anxiety measures. Cella D, } \\
\text { Choi SW, Condon DM, Schalet B, Hays RD, Rothrock NE, } \\
\text { et al. PROMIS adult health profiles: efficient short-form } \\
\text { measures of seven health domains. Value Health } 2019 \text {; } \\
\text { 22(5):537-544. }\end{array}$} \\
\hline
\end{tabular}

9 months preceding March 2020, 166 performed three SSEs. Subjects were $54.5 \%$ female (115 of 211), had a mean age of 55 years, and $78.7 \%$ (166) had regularly scheduled appointments for skin examinations. All studied melanoma SSE anxiety topics increased during COVID -19 and there was a statistically significant difference in SSE anxiety for 3 self-reported responses prior to and during COVID-19 (Table 1). 
Table 2. Comparison of mole self-sampling specimens with physician provided samples

\section{A) PLA patient self-sampling under the remote supervision of a licensed healthcare professional}

\begin{tabular}{|c|c|c|c|c|c|c|}
\hline Sample \# & Sex & $\begin{array}{c}\text { Lesion } \\
\text { Size }(\mathrm{mm})\end{array}$ & $\begin{array}{c}\text { Lesion } \\
\text { Location }\end{array}$ & LINC & PRAME & PLA \\
\hline Sample 1 & $\mathrm{M}$ & $5 \times 5$ & Hip & ND & ND & N \\
\hline Sample 2 & $\mathrm{F}$ & $5 \times 5$ & Shoulder & ND & ND & N \\
\hline Sample 3 & $\mathrm{M}$ & $7 \times 9$ & Leg & ND & ND & N \\
\hline Sample 4 & $\mathrm{M}$ & $7 \times 7$ & Scalp & ND & ND & N \\
\hline Sample 5 & $\mathrm{F}$ & $7 \times 8$ & Back & ND & ND & N \\
\hline Sample 6 & $\mathrm{F}$ & $6 \times 11$ & Back & ND & ND & N \\
\hline Sample 7 & F & $7 \times 7$ & Back & ND & ND & N \\
\hline
\end{tabular}

\begin{tabular}{|c|c|c|c|c|c|c|}
\hline B) $\mathbf{F}$ & \multicolumn{6}{|c|}{ 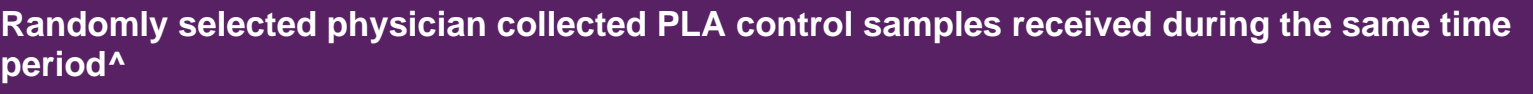 } \\
\hline Sample \# & Sex & $\begin{array}{l}\text { Lesion } \\
\text { Size }(\mathrm{mm})\end{array}$ & $\begin{array}{l}\text { Lesion } \\
\text { Location }\end{array}$ & LINC & PRAME & PLA \\
\hline Control 1 & $M$ & $10 \times 10$ & Back & ND & ND & $\mathrm{N}$ \\
\hline Control 2 & $\mathrm{~F}$ & $9 \times 10$ & Ankle & QNS & QNS & QNS \\
\hline Control 3 & $\mathrm{~F}$ & $10 \times 7$ & Back & ND & $\mathrm{D}$ & $\mathrm{P}$ \\
\hline Control 4 & $\mathrm{~F}$ & $6 \times 7$ & Leg & ND & ND & $\mathrm{N}$ \\
\hline Control 5 & $\mathrm{~F}$ & $11 \times 12$ & Back & ND & ND & $\mathrm{N}$ \\
\hline Control 6 & $\mathrm{~F}$ & $11 \times 13$ & Buttock & ND & ND & $\mathrm{N}$ \\
\hline Control 7 & $M$ & $6 \times 4$ & Flank & $\mathrm{D}$ & ND & $\mathrm{P}$ \\
\hline Control 8 & $\mathrm{~F}$ & $6 \times 5$ & Breast & ND & $\mathrm{D}$ & $P$ \\
\hline Control 9 & $\mathrm{~F}$ & $12 \times 11$ & Back & ND & ND & $\mathrm{N}$ \\
\hline Control 10 & $M$ & $13 \times 11$ & Back & ND & ND & $N$ \\
\hline
\end{tabular}

Abbreviations: Pigmented Lesion Assay (PLA), Long Intergenic Non-Coding RNA 518 / LINC00518 (LINC), Preferentially Expressed Antigen in Melanoma (PRAME), Male (M), Female (F), Quantity Not Sufficient (QNS), Detected (D), Not Detected (ND), Positive (P), Negative (N), Same Patient. *\$

`Samples collected in states without restricted physician access.

After COVID-19, five subjects noted change in a mole. Subjects related that lack of physician access influenced subjects' decision to do mole self-sampling. Guided self-sampling led to successful molecular risk factor analyses by PLA in $7 / 7(100 \%)$ of cases compared to $9 / 10(90 \%)$ randomly selected physician-sampled control cases received during the same timeframe (Table 2).

\section{DISCUSSION}

In this study, increased SSE anxiety may be partially attributed to generalized health anxiety; however, lack of physician access to provide skin examinations for melanoma survivors with self-identified concerning moles was an important factor in patients' desire for high-quality alternatives. A limitation was lack of general anxiety assessment. This research demonstrates

May 2020 Volume 4 Issue 3 
that adhesive patch self-sampling under physician guidance is a viable specimen collection option. Remote collection can expand access to assessment by PLA, a gene expression - based melanoma rule-out test with a negative predictive value above $99 \%$, that reduces avoidable surgical biopsies of pigmented lesions clinically suspicious for melanoma by over $90 \%$ as demonstrated in a recent registry study of 3,418 cases. $^{6}$

\section{CONCLUSION}

This proof-of-concept research demonstrates that patients are able to reliably perform selfsampling of concerning moles under remote physician supervision. Patient-obtained skin sample collection using adhesive patches was successful in $100 \%$ of cases enabling actionable molecular pathology PLA test reports to rule-out melanoma in all cases. The offered teledermatology solution provided pigmented lesion management with a negative predictive value of over $99 \%$ and reduced patient anxiety while avoiding office visits during a period when office visits are limited by COVID-19 to essential care. Adhesive patch self-sampling under remote clinician guidance is a viable specimen collection option.

Acknowledgment: Supported by R01 CA154908 to June K. Robinson, MD, from the United States National Cancer Institute. Pigmented lesion assay testing provided by DermTech Inc. (La Jolla, CA). Mary Kwasny, PhD, Department of Preventive Medicine, Northwestern University, Feinberg School of Medicine, Chicago, IL, provided statistical analysis and received compensation for her role.

Conflict of Interest Disclosures: Dr. Jansen is an employee of DermTech, Inc. Dr. Robinson has no conflicts of interest.

Clinical Trials registration: NCT02854657

Corresponding Author:
June K. Robinson, MD

Department of Dermatology

Northwestern University Feinberg School of Medicine

645 N Michigan Ave, Suite 1050

Chicago, IL, 60611 USA

Email: june-robinson@northwestern.edu

\section{References:}

1. Robinson JK, Wayne JD, Martini MC, Hultgren BA, Mallett KA, Turrisi R. Early detection of new melanomas by patients with melanoma and their partners using a structured skin self-examination skills training intervention: a randomized clinical trial. JAMA Dermatol. 2016;152(9):979-985.

2. President Trump Expands Telehealth Benefits for Medicare Beneficiaries During COVID-19 Outbreak. March 17, 2020.

https://www.cms.gov/newsroom/pressreleases/president-trump-expands-telehealthbenefits-medicare-beneficiaries-during-covid-19outbreak

3. de Carvalho TM, Noels E, Wakkee M, Udrea A, Nijsten T. Development of smartphone apps for skin cancer risk assessment: progress and promise. JMIR Dermatol. 2019;2 (1):e13376.

4. Gerami P, Yao S, Polsky D, et al. Development and validation of a noninvasive 2-gene molecular assay for cutaneous melanoma. J Am Acad Dermatol. 2017;76:114-20.

5. Robinson JK, Reavy R, Mallett KA, Turrisi R. Remote partner assisted skin self-examination skills training of melanoma survivors and their partners. Australian J Dermatol. 2019;60 (1):e8082.

6. Brouha B, Ferris LK, Skelsey MK, et al. Realworld utility of a non-invasive gene expression test to rule out primary cutaneous melanoma: a large US registry study. J Drugs Dermatol. 2020 March, 19 (3). 